\title{
Active Semiconductor Nanophotonics based on Deterministic Quantum Wire and Dot Systems
}

\author{
Pascal Gallo $^{1 *}$, K.Atlasov ${ }^{1}$, M. Calic ${ }^{1}$, B. Dwir ${ }^{1}$, M. Felici ${ }^{1 \mathrm{a}}$, F. Karlsson ${ }^{2}$, A. Mohan ${ }^{1}$, E. Pelucchi ${ }^{3}$,

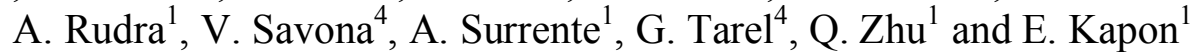 \\ ${ }^{1}$ Ecole Polytechnique Fédérale de Lausanne, Laboratory of Physics of Nanostructures \\ 1015 Lausanne, Switzerland \\ 2 IFM Material Physics Linköping University S581 83, Sweden \\ ${ }^{3}$ Tyndall National Institute "Lee Maltings" Prospect Row, Cork, Ireland \\ ${ }^{4}$ Ecole Polytechnique Fédérale de Lausanne, Laboratory of Theoretical Physics 1015 Lausanne, \\ Switzerland
}

\begin{abstract}
We investigate the use of MOVPE-grown ordered nanostructures on non-planar substrates for quantum nano-photonics and quantum electrodynamics-based applications. The mastering of surface adatom fluxes on patterned GaAs substrates allows for forming nanostrucutres confining well-defined charge carrier states. An example given is the formation of quantum dot (QD) molecules tunneled-coupled by quantum wires (QWRs), in which both electron and hole states are hybridized. In addition, it is shown that the high degree of symmetry of QDs grown on patterned (111)B substrates makes them efficient entangled-photons emitters. Thanks to the optimal control over their position and emission wavelength, the fabricated nanostructures can be efficiently coupled to photonic nano-cavities. Low-threshold, optically pumped QWR laser incorporating photonic crystal $(\mathrm{PhC})$ membrane cavities are demonstrated. Moreover, phononmediated coupling of QD exciton states to PhC cavities is observed. This approach should be useful for integrating more complex systems of QWRs and QDs for forming a variety of active nano-photonic structures.
\end{abstract}

Keywords: Quantum dots, quantum wires, photonic cavities, photonic crystals, MOVPE, nanostructures

\section{INTRODUCTION}

Incorporating semiconductor quantum nanostructures such as quantum wires (QWRs) and quantum dots (QDs) into nano-photonic elements provides means for exploring both fundamental quantum photonics phenomena and applications in new photonic devices and systems. Examples include solid-state cavity quantum electrodynamics and quantum information processing and communications ${ }^{1,2}$. Most work in this field has been done using self-assembled quantum nanostructures ${ }^{3,4,5,6,7,8}$, which, however, do not offer the necessary control in terms of optical spectra and positioning of the QWRs/QDs on the photonic chip.

Here, we review recent progress in quantum nano-photonics based on ordered $Q W R$ and $Q D$ structures grown on patterned substrates using metallorganic vapor phase epitaxy (MOVPE) ${ }^{9}$. In particular, we discuss V-groove QWRs and pyramidal QDs formed due to growth rate anisotropy and nano-capillarity on nonplanar, patterned GaAs substrates ${ }^{10}$. This approach yields high quality, site-controlled (In)GaAs/(Al)GaAs QWR and QD systems, e.g., regular arrays of pyramidal QDs with $\sim 1 \mathrm{meV}$ inhomogeneous broadening ${ }^{11}$. The confining potential of these wires/dots can be shaped by

\footnotetext{
${ }^{a}$ Now at Sapienza Universita di Roma, Piazzale Aldo Moro 2, 00185 Roma, Italy

*e-mail : pascal.gallo@epfl.ch
}

Active Photonic Materials IV, edited by Ganapathi S. Subramania, Stavroula Foteinopoulou, Proc. of SPIE Vol. 8095, 80950Y - (C) 2011 SPIE · CCC code: 0277-786X/11/\$18 · doi: 10.1117/12.895142 
thickness and/or composition adjustment, producing structures of elaborated potential distributions, e.g., QD molecules and superlattices ${ }^{12}$. The intrinsically high symmetry of the pyramidal QDs yields unique excitonic features such as vanishing fine structure splitting (FSS), permitting efficient generation of polarization-entangled photons ${ }^{13}$.

Based on these ordered QWR/QD systems, several nano-photonic devices have been conceived and studied. These include coupled photonic crystal $(\mathrm{PhC})$ cavities showing coupled-mode loss splitting ${ }^{14}$, optically pumped, $\sim 1 \mu \mathrm{m}$ long PhC-QWR lasers with sub- $\mu \mathrm{W}$ threshold and coupled-cavity PhC-QWR lasers ${ }^{15}$, and single-QDs integrated with $\mathrm{PhC}$ membrane cavities ${ }^{16}$. More recently, deterministic coupling of single pyramidal QDs in PhC membrane cavities has allowed the identification of phonon-mediated coupling of the QD exciton and the cavity mode, which persists only for limited (few meV) cavity detuning ${ }^{17}$. This paves the way to the deterministic coupling of several QD-excitons via cavity photons, interesting for exploring many-body quantum photonics phenomena ${ }^{18}$.

Some details of the technology, physics and applications of the resulting QWR and QD systems and their integration with photonic elements are described in this review.

\section{MOVPE OF QUANTUM NANOSTRUCTURES ON NON-PLANAR SUBSTRATES}

MOVPE on patterned GaAs substrates is an efficient and reproducible method for producing site and energy-controlled nanostructures ${ }^{9}$. The fundamental mechanisms involved are three-fold. First, precursors have to decompose in order to release adatoms that will contribute to crystal growth. The decomposition rate depends on the crystallographic facet orientation. Second, surface adatoms, released via precursor decomposition and, driven by capillarity, diffuse towards regions in the recesses where curvature is higher. Finally, the sticking efficiency, that is, the incorporation rate in the semiconductor matrix, depends as well on the facet orientation. Hence, on patterned surfaces, one can use these parameters in order to direct adatom fluxes towards desired locations on a substrate, thus forming nanostructures with the desired properties ${ }^{10}$. In particular, if one grows material on (111)B GaAs substrate patterned with pyramidal recesses with (111)A facets, decomposition of precursors will only occur on the latter facets, releasing adatoms mainly inside the pyramids. Thanks to capillarity, adatoms accumulate along the edges of grooves or at the apex of the recesses, as shown in figure 1. If the bandgap of the deposited material is lower than the host matrix, charge carriers can thus be confined in the QWRs and the QDs thus formed, respectively ${ }^{19}$. This forms the basis for producing systems of quantum-confined nanostructures with well-defined, low dimensional potential barriers.

Moreover, one can envisage using the differential diffusion length of group III elements on the patterned facets, when growing a ternary alloy such as AlGaAs, as another degree of freedom for forming heterostructures with complex potential distributions ${ }^{20}$. Figure 2 shows an example of stacked QDs formed along a central channel of lower bandgap $\mathrm{AlGaAs}$ grown in a GaAs pyramidal recess. The longer diffusion of $\mathrm{Ga}$ adatoms as compared with $\mathrm{Al}$ ones, combined with capillarity effects, produces a central low bandgap channel, referred to as the vertical QWR (VQWR), richer in Ga. Along this channel, one can modulate the potential by simply modulating the nominal Al content during growth, forming QDs with very well controlled composition and geometry.

a)

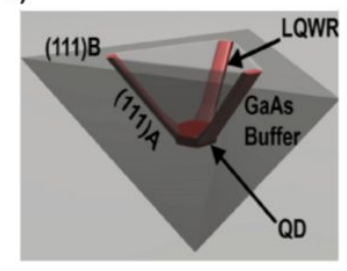

b)

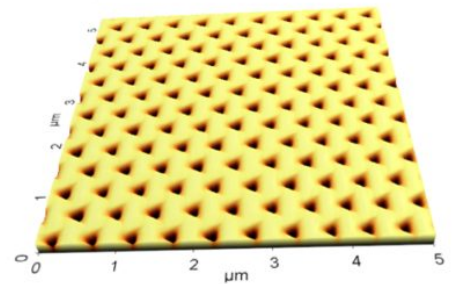

c)

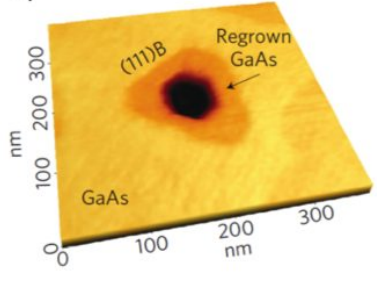

Figure 1. (a) 3D schematic viewgraph of a pyramidal recess into which a GaAs buffer and a GaInAs layer were subsequently grown. The structures formed are three lateral quantum wires (LQWRs) and a quantum dot (QD). (b) AFM image of an ordered ensemble of QDs grown on a $500 \mathrm{~nm}$ pitch array of pyramidal recesses. ${ }^{11}$ (c) High resolution AFM of a single QD, exhibiting a hexagonal symmetry. ${ }^{13}$ 

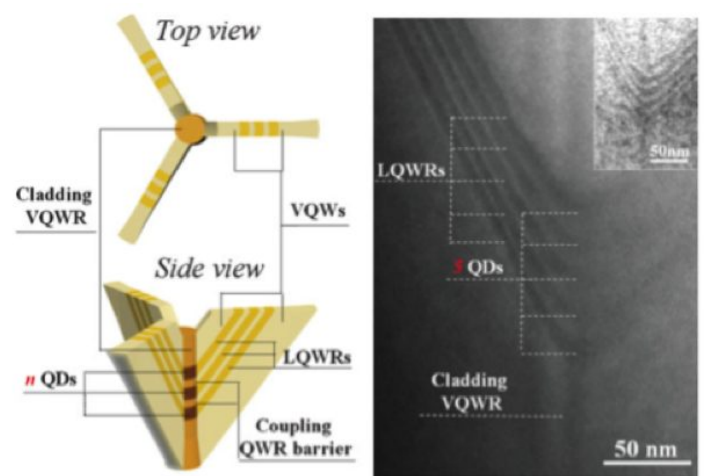

Figure 2. Ternary AlGaAs alloy heterostructure grown in a pyramidal recess, forming a vertical stack of QDs tunnelcoupled by QWR barriers. ${ }^{12}$

This method is therefore a versatile way for producing nanostructures starting from the simplest configuration, e.g., a single QD with bulk 3D barriers, to very elaborate stacked QDs connected with 1D barriers. Since both position and emission wavelength are precisely determined, one can insert these nanostructures in photonic cavities, forming devices that allow studying fundamental quantum electrodynamics effects and potentially applicable in quantum information technology.

\section{PYRAMIDAL QDS AS EFFICIENT ENTANGLED PHOTONS EMITTERS}

One striking feature of the pyramidal QDs is their high degree of $\mathrm{C}_{3 \mathrm{v}}$ symmetry (figure $1 \mathrm{a}$ )), brought about by the growth on the (111)B-oriented substrate ${ }^{21}$. This is unlike growth of self-assembled Stranski-Krastanow QDs on (100 substrates), which show lower symmetry in the growth plane.

This structural symmetry also results in higher symmetry of the excitonic states, which, is of particular interest for producing entangled pairs of photons in the $2 \mathrm{X}-\mathrm{X}$ radiative cascade process ${ }^{13}$ (see Fig. 3b). Indeed, the excitonic fine structure splitting (FSS), i.e., the difference in the X energy for two orthogonal polarization of the emitted photons measured in the substrate plane, vanishes in QDs presenting at least a 3-fold symmetry in both their macroscopic and microscopic structure. The two paths of the radiative cascade decay of the $2 \mathrm{X}$ state are then exactly undistinguishable, yielding polarization-entangled pairs of photons.

a)

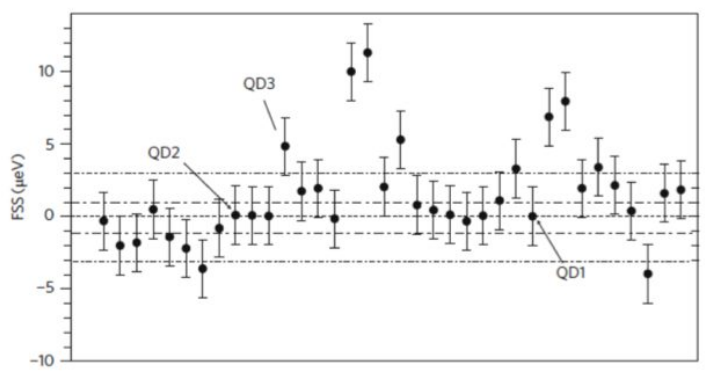

b)

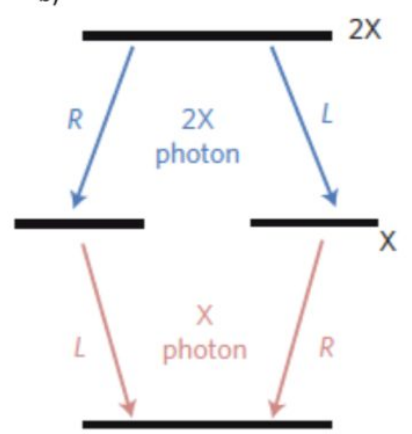

Figure 3. Pyramidal QDs as efficient entangled photons emitters. Fine structure splitting for a group of single QDs (a); and the identical paths in the XX-X radiative cascade used for the production of the polarization entangled photons(b). ${ }^{13}$ 
As witnessed by the AFM picture of a grown pyramid presented in figure 1, the symmetry of the structure is 6-fold, which, combined with the 3 -fold $\mathrm{C}_{3 \mathrm{v}}$ symmetry of the crystal, explains why the FSS values measured are smaller than $3 \mu \mathrm{eV}$ in $80 \%$ of the pyramidal InGaAs/GaAs QDs tested (figure $3 \mathrm{a}$ )). To achieve comprehensive characterization of the polarization entanglement, a quantum state tomography set-up ${ }^{22}$ was used to construct the full two-photon polarization density matrix from a linear combination of cross-correlation measurements performed using 16 different polarization combinations. Moreover, we were able to demonstrate that several entanglement criteria such as fidelity, concurrence and the Peres criterion were satisfied for QDs 1 and 2 in figure 3a, unlike QD 3 ; we therefore established a limit for the FSS of $3 \mu \mathrm{eV}$, that allows the production of entangled pairs of photons, which holds for $80 \%$ of our QDs.

\section{TAILORING AND COUPLING QUANTUM STATES IN PYRAMIDAL QDS}

Physical implementation of quantum computers ${ }^{23}$ is still a subject of intensive research ${ }^{24}$. The best candidates up to now remain semiconductor-based devices, especially QD-confined exciton states serving to represents the Q-bits ${ }^{25}$. Here, we demonstrate engineering of conduction and valence bands of pyramidal QDs, allowing for producing excitons emitting photons with the desired polarization. Further more, building logic gates requires coherent interaction between excitons; in this direction, we demonstrate the possibility to do so through tunnel coupling of closely stacked pyramidal QDs in QD-molecule structures.

\subsection{Engineering conduction and valence bands in Pyramidal QDs}

The growth mechanisms of pyramidal QDs are based on capillarity of surface adatoms; a particular growth regime is reached when the size of the pyramidal recesses becomes comparable to the adatoms diffusion length. In that case, the size of the QDs, especially their height, highly depends on the size of the host recess (figure 4a). Through this mechanism, one can tune the hole character of the ground state transition, and therefore the polarization of the photons emitted by the confined excitons, by changing the pyramid size ${ }^{26}$. Figure 4(b) presents the integrated intensity of the ground state transition measured as a function of the angle of the rotated analyzer in the yz plane for three different InGaAs/GaAs QDs. For a system that is isotropic in linear polarization in the plane perpendicular to $z$, the intensity of light linearly polarized perpendicular to z implies a purely heavy-hole like character, light polarized parallel to $z$ implies a purely light-hole like character, and an isotropic emission implies complete mixing of the hole states. For QDs formed in a pyramid of $250 \mathrm{~nm}$ side the emission is polarized perpendicular to $\mathrm{z}$, whereas for $395 \mathrm{~nm}$ side pyramid the emission is polarized parallel to the $\mathrm{z}$ axis. For $325 \mathrm{~nm}$ side pyramid, polarization of the emission is almost isotropic. This implies a clear switching of the ground state hole character, from a predominantly light-hole like character for QDs grown in the smaller pyramids of $250 \mathrm{~nm}$ side (thicker dots) to a predominantly heavy-hole like character for QDs grown into bigger pyramids of $395 \mathrm{~nm}$ side (thinner dots).

a)

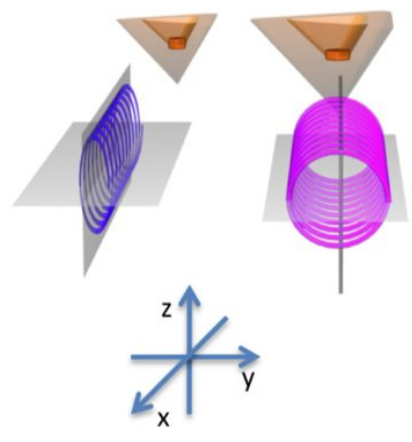

b)

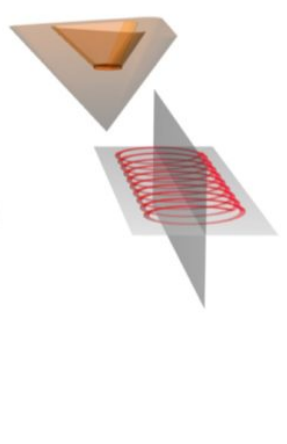

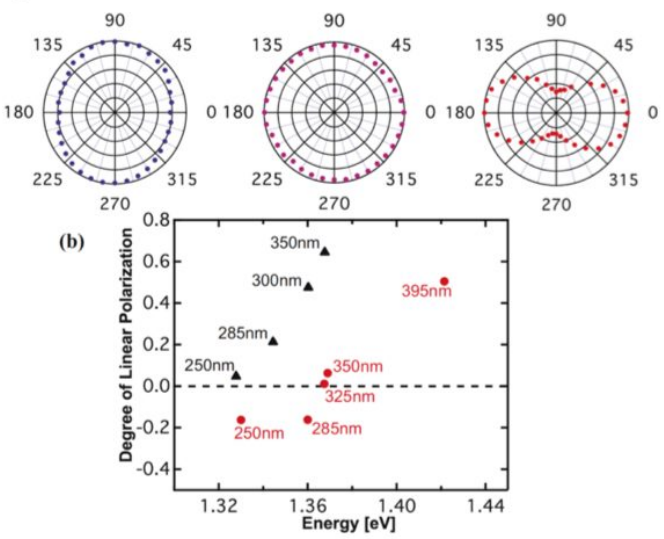

Figure 4. On the same sample, varying the pyramid size, one can tune the aspect ratio of the QDs thanks to the modulation of capillarity effects which become dominant as the pyramid size is reduced below the adatom surface diffusion length (a). As a consequence, polarization of the photons emitted from the edge of the sample can be selected and prepared, as depicted by the linear polarization dependence of the ground state of three different QDs of structure B, grown into pyramids with sides of $250 \mathrm{~nm}, 325 \mathrm{~nm}$ and $395 \mathrm{~nm}$ (from left to right) in the $y z$ plane (b). The measured degree of linear polarization for structure A (triangles) $\left(\operatorname{In}_{0.3} \mathrm{Ga}_{0.7} \mathrm{As}\right.$ QD layer with nominal thickness of $0.6 \mathrm{~nm}$ ) and structure B (circles) (same except for the QD composition, $\operatorname{In}_{0.2} \mathrm{Ga}_{0.8} \mathrm{As}$ ) is depicted for various QDs grown into pyramids of different side lengths (c). ${ }^{26}$ 


\subsection{Tunnel-coupling of excitons in pyramidal QD molecules}

We now focus on more elaborate structures comprising QDs connected with QWRs. ${ }^{12}$ These structures are the result of growth of AlGaAs heterostructures in the pyramidal recesses. The Ga-rich vertical QWR formed at the center of the pyramid serves as the backbone of an attached QD structure with QWR barriers. The dimensions and composition of the QDs and QWR barriers can be adjusted by proper modulation of the Al content during growth. The QWR barriers serve to better couple the confined states in adjacent QDs due to lateral confinement of the wavefunctions involved.

A proof of the tunnel coupling between the QDs, especially the hole states, can be found in figure 5. On the left hand side of the panel, several QD structures formed by vertical stacking of pyramidal QDs are shown. The second and third parts of the panel shows the calculated wavefunction isosurfaces for the electron and the hole states, and the related emission spectra. The problem was treated within the framework of the envelope function approximation, the conduction band was described by a single band, whereas the valence band was described by $4 \times 4$ Luttinger Hamiltonian, taking into account mixing between light and heavy holes ${ }^{27}$. The main effect of the hybridization of the hole states in the 2-QD and the 3-QD molecules is to render the ground valence band state light-hole like, in contrast to the case of the single QD, in which it is heavy-hole like. The ground state transition thus provides an efficient way to characterize the coupling between the vertically stacked QDs. Indeed, the measured spectra in Fig. 5(d) exhibit an evolution from light polarized mainly perpendicular to the vertical QWR axis, for a single QD, to light polarized mainly along the vertical QWR axis for the QD molecules. This demonstrates the hybridization of the hole states in the QD molecules, which is very difficult to achieve with self-assembled dots in which size variations induced localized carrier states in different parts of the molecule.

a)

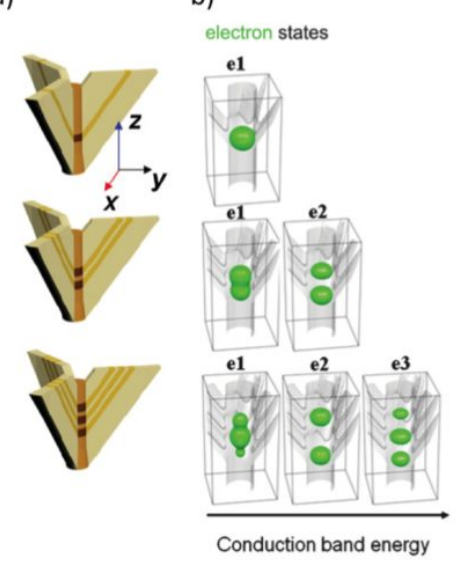

b)

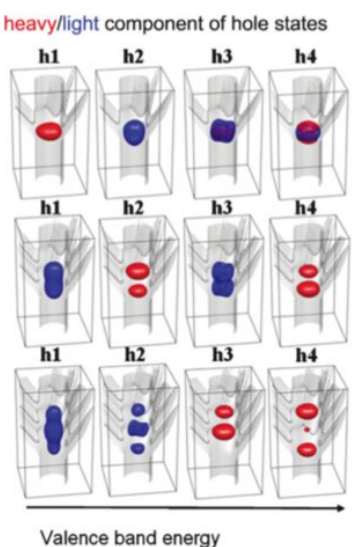

d)

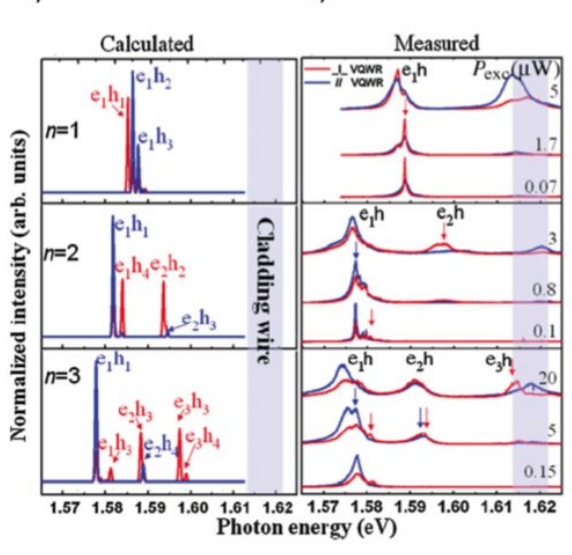

Figure 5. (a) Sketch of one, two and three QDs grown in pyramidal recesses. The isosurface of their wavefunction amplitude is calculated and depicted in (b) for both electron and hole states. Wavefunction hybridization and corresponding changes in the hole character of the ground states predicted by calculations (c), in very good agreement with the measured photoluminescence spectra $(\mathrm{d}) .^{12}$ 


\section{COUPLING QWR AND QD STATES TO PHOTONIC NANOCAVITY MODES}

A goal that is as rewarding as challenging is to couple excitonic states confined in nanostructures to photonic modes of optical nanocavities. The interest here is two-fold; for the realization of low threshold lasers, one can benefit from enhanced light-matter interaction in photonic cavities to decrease the threshold. For the conversion of stationary Q-bits, which would be implemented on the spin of the excitons trapped in a QD, into flying Q-bits implemented on photons, one can benefit from Purcell enhancement of spontaneous emission to decrease the radiative time of the excitons and therefore decrease the probablity for the exciton spin to dephase $\mathrm{e}^{28}$. Here, we investigate both goals respectively with sitecontrolled QWRs and QDs integrated in photonic crystal slab cavities.

\subsection{Phonon-mediated coupling of Pyramidal QDs to photonic crystal nanocavities}

A major advantage of pyramidal QDs is the control of both their nucleation site and emission wavelength. This allows for a deterministic coupling to PhC cavities ${ }^{16,17}$, as described in figure 6. In this case, the pitch of arrays of ordered QDs, whose positions are precisely known, is exactly twice that of the PhC lattice. Hence, when etching the PhCs, the QDs are all removed except for the very QD that is to be integrated in the $\mathrm{PhC}$ cavity, as shown in figure 6a. A scanning electron microscope (SEM) image of the resulting sample is presented in figure 6b; a residual hole marks the presence of the QD, which alignment allows it to be exposed to at least $60 \%$ of the maximum optical field intensity. Since the emission energy of the QDs can be controlled, it can be matched with the cavity resonance, allowing for an effective coupling between the QD exciton and the cavity mode.
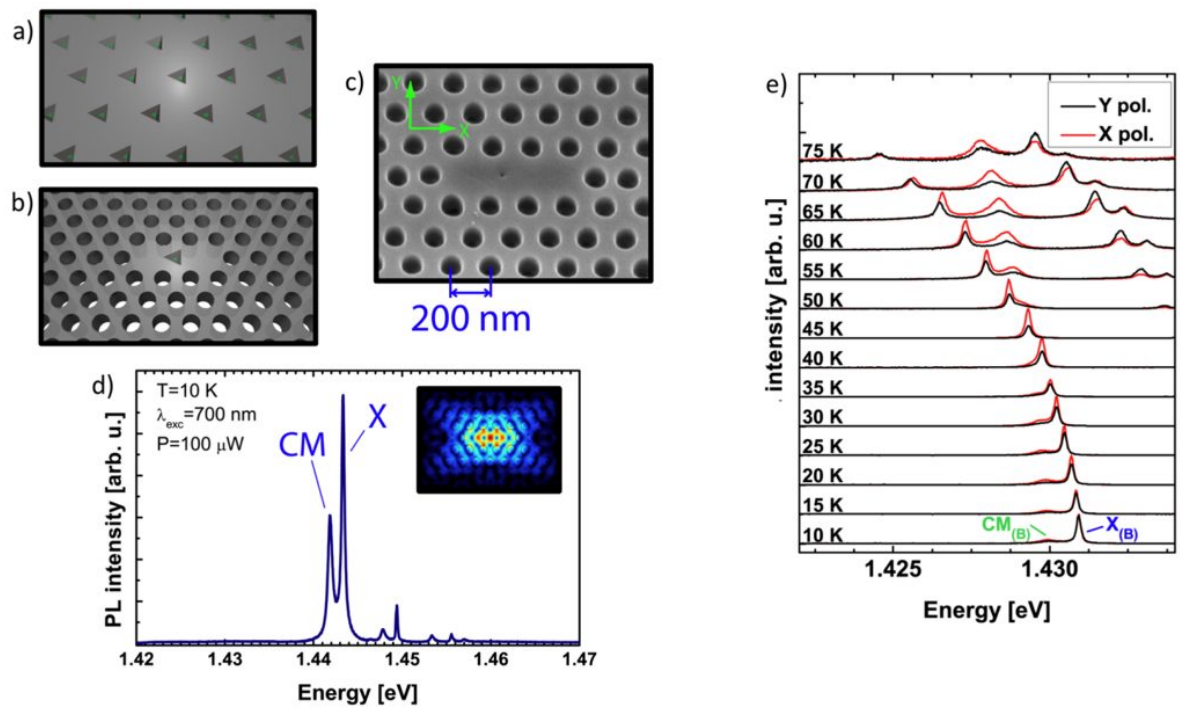

Figure 6. (a) Sketch of the ensemble of pyramidal QDs used in the experiments. (b) Schematic illustration of a single-QD nanocavity device. (c) SEM image of an actual device (tilted view). (d) Typical low-temperature PL spectrum of the structure. ${ }^{17}$

Evidence of coupling between the QD and the cavity is presented in figure 6c, where a typical spectrum emitted by the structure is displayed. One can clearly distinguish both peaks attributed, respectively, to the excitonic transition of the QD and the photons emitted by the cavity mode. In order to get a deeper insight into the coupling between the QD and the cavity, the excitonic transition was progressively tuned into resonance through temperature change, thanks to the weaker dependence on temperature of the cavity energy. For each temperature, the linear polarization along and 
perpendicular to the cavity axis is resolved in the emission spectra. It is clearly visible that the polarization of the QD progressively acquires that of the cavity, within a characteristic detuning range of approximately $2 \mathrm{meV}$. This result is striking, since it appears to be in contrast with other experiments involving SK QDs, for which the off-resonant coupling is seen over much larger detunings (we designate this as far off-resonant (FOR) coupling) ${ }^{29}$. The FOR coupling is in the latter case induced by multi-excitonic transitions provided by the hybridization of QDs states with the 2D sates of the wetting layer ${ }^{30}$. This effect is detrimental since for QED applications, one must be able to couple single excitons to a cavity mode in a deterministic way, for example when conversion of an excitonic Q-bit into a photonic single Q-bit is envisaged. Theoretical modeling allowed us to demonstrate that only phonon-mediated processes are involved in the offresonant coupling of an exciton confined in a pyramidal QD with a cavity mode (CM); these processes have a characteristic energy of less than $2 \mathrm{meV}$ at low temperatures, which permits to have a single excitonic feature coupled to a cavity mode ${ }^{17}$.

A direct consequence of the coupling is manifested in the corresponding second-order photon correlation function. Figure 7 shows the correlation histograms $\mathrm{g}^{(2)}(\tau)$ acquired for the $\mathrm{X}$ and $\mathrm{CM}$ lines of pyramidal QD-nanocavity system at a detuning of $\sim 1.5 \mathrm{meV}$. The observation of antibunching in the X-X and CM-CM auto-correlations, as well as in the CM-X cross-correlation, unambiguously proves that the coupling between the pyramidal QD and the cavity mode is regulated at the level of single photons and that also the cavity emission takes place in the quantum regime. Previous reports of similar experiments ${ }^{30}$, but with self-assembled QDs, revealed that the photons emitted by the cavity exhibited classical correlations, which is an artifact originating from FOR coupling occurring in that case.

These results prove that FOR is not an intrinsic property of semiconductors, and that one can transpose the clean experiments performed with single atoms in photonic cavity to solid state using PQDs. Some of the concerns that have been brought about by the physical implementation of quantum information processing in semiconductors are thus addressed.

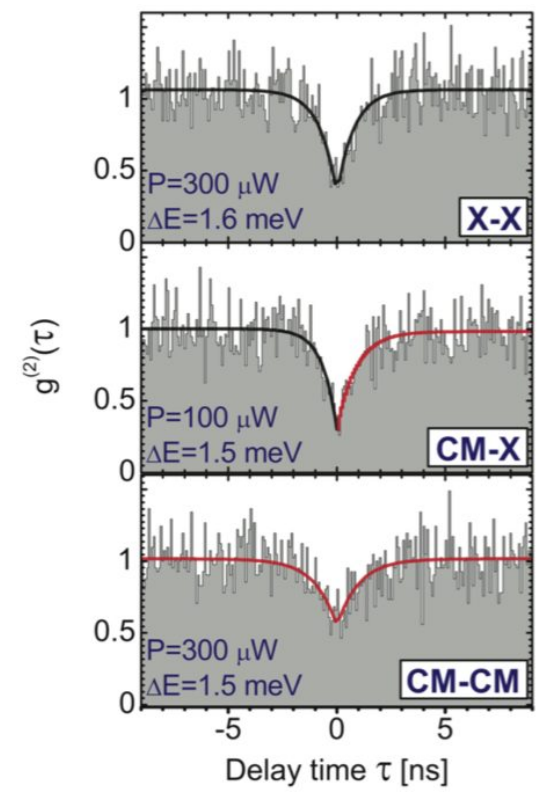

Figure 7. Second-order correlation histograms for a QD-cavity system for $1.5 \mathrm{meV}$ detuning, exhibiting antibunching in the $\mathrm{X}-\mathrm{X}$ and CM-CM auto-correlations, as well as in the CM-X cross-correlation.

\subsection{Low-threshold lasers with QWRs integrated in photonic nanocavities}

Integrating low-dimensional nanostructures in photonic cavities with very low mode volume is of great interest for realizing ultra low-threshold, low power consumption and high modulation speed lasers. Indeed, conventional lasers 
using QWs in large mode volume cavities suffer from losses due to spontaneous emission into other modes than the lasing ones, as well as very low Purcell enhancement of spontaneous emission because of the poor spatial overlap of the gain medium with the optical field and the large mode volume of the cavity. In turn, the lasing threshold is significantly increased with respect to the ideal case.

We realized optically pumped QWR-based lasers employing PhC nanocavities ${ }^{15}$. Growth of the InGaAs/GaAs QWRs was performed with MOVPE on V-groove-patterned (100) GaAs substrates. The growth mechanisms are similar to the ones described in the first section, except that the preferential accumulation of material at the bottom of the grooves thanks to capillarity leads to 1D nanostructures, whose cross-section is displayed in figure 8 , showing in this case 3 stacked QWRs. Since the position and emission energy can be precisely controlled, one can align these structures with a $\mathrm{PhC}$ cavity which resonance is determined by the periodicity and size of the drilled holes. An AFM image of the resulting structure is shown in figure 8. The gain medium made of 3 stacked QWRs is largely overlapping the optical field of the L6 PhC cavity, whose mode volume is very small $\left(2 \lambda^{3}\right)$. The large mode wavelength separation allows for efficient coupling to a single cavity mode.

A first indication that the device lases is given by the time resolved measurements of the optical response of the optically pumped device presented in figure 8. For low excitation powers, the decay can be fitted with a single exponential, corresponding to the spontaneous emission process. As the power is increased, a second exponential decay slope appears with a shorter characteristic time, corresponding to stimulated emission. The estimated threshold is approximately $240 \mathrm{nW}$ (absorbed power), suggesting that the spontaneous coupling constant is very large; indeed, it could be extracted from power dependant light in-light out measurements that $\beta=0.3$ for this device, to be compared to devices employing QWs in large mode volume cavities whose $\beta$ typically ranges from 0.01 to 0.0001 .

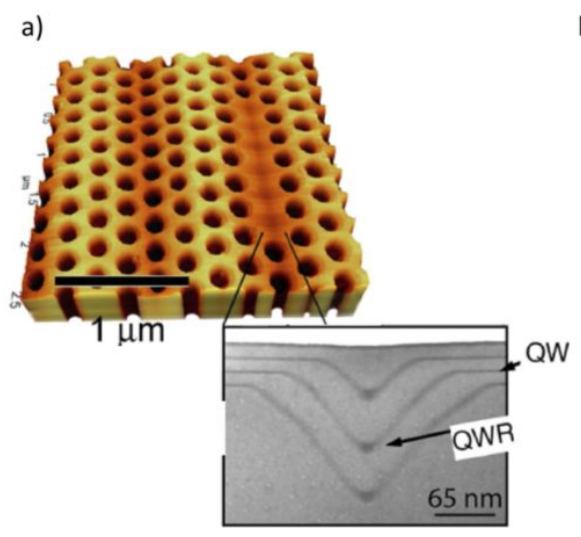

b)

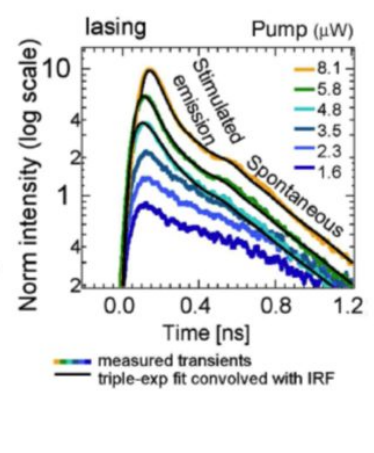

c)

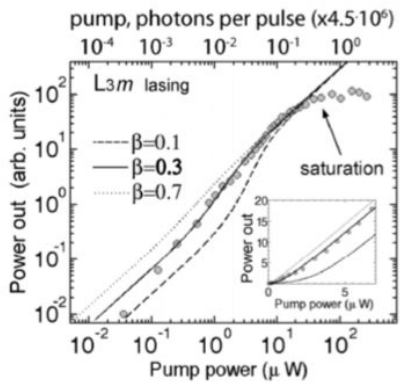

Figure 8. Three stacked GaInAs quantum wires grown on V-groove patterned (100) GaAs surface were incorporated in L6 cavities, as witnessed by the AFM picture (a). Thanks to Purcell enhancement of emission processes, quantum wires which emission energy matched larger-Q cavities exhibited stimulated emission, as witnessed by the timeresolved spectra (b) which could be fitted with two exponentials, corresponding respectively to the stimulated and spontaneous emission. (c) Power dependence of input light vs output light, from which a high spontaneous coupling constant $\beta$ of 0.3 could be determined. ${ }^{15}$

\section{CONCLUSION}

We demonstrated the interest of using MOVPE-grown nanostructures on non-planar substrates for applications in quantum nano-photonics. In particular, the resulting QD structures are versatile, allowing for precise engineering of carriers wavefunction configuration and energy, and exhibit a higher degree of structural and electronic states symmetry than most self-assembled QDs. Several examples of these site controlled nanostrcutures and their optical properties have been described, including emitters of polarization entangled photons, QDs with a controlled degree of valence band 
mixing and polarization of emitted photons, QD molecules showing hybridization of valence band states, QDs integrated with PhC cavities, and low power consumption QWR-PhC lasers. These nanostrcture systems should find applications in various physical implementations for quantum information technology.

\section{REFERENCES}

${ }^{1}$ See, e.g, J.L. Obrien, A. Furusawa and J. Vuckovic, Nature Photonics 3, 687 (2009).

${ }^{2}$ D.P. DiVincenzo, Fortschritte der Physik 48, 771-784 (2000)

${ }^{3}$ J. P. Reithmaier et al., Nature 432, 197 (2004).

${ }^{4}$ T. Yoshie et al., Nature 432, 200 (2004).

${ }^{5}$ E. Peter et al., Phys. Rev. Lett. 95, 4 (2005).

${ }^{6}$ G. Khitrova et al., Nat. Phys. 2, 81 (2006).

${ }^{7}$ M. Nomura et al., Nat. Phys. 6, 279 (2010).

${ }^{8}$ B. Ellis et al. Nat. Photonics 5, 297 (2011).

${ }^{9}$ E. Kapon et al., Appl. Phys. Lett. 50, 347 (1987).

${ }^{10}$ G. Biasiol and E. Kapon, Phys. Rev. Lett. 81, 2962 (1998).

${ }^{11}$ A. Mohan et al., Small 6, 1268 (2010).

${ }^{12}$ Q. Zhou et al., Small 5, 329 (2009).

${ }^{13}$ A. Mohan et al., Nature Photonics, 4, 302 (2010).

${ }^{14}$ K. Atlasov et al., Optics Express 16, 16255 (2008).

${ }^{15}$ K. Atlasov et al., Optics Express 17,18178 (2009); K. Atlasov et al., Optics Express (2011).

${ }^{16}$ P. Gallo et al., Appl. Phys. Lett. 92, 63101 (2008).

${ }^{17}$ M. Calic et al. Phys. Rev. Lett. 106, 227402 (2011). 
${ }^{18}$ M.J. Hartmann, F.G.S.L. Brandao and M.B. Plenio, Laser\&Photon. Rev. 2, 527 (2008).

${ }^{19}$ A. Hartmann et al., J. Phys. Condens. Matter 11, 5901 (1999).

${ }^{20}$ Q. Zhu et al., Phys. Rev. B, 82, 165315 (2011)

${ }^{21}$ F. Karlsson et al. Phys Rev. B 81, 161307 (2010).

${ }^{22}$ D.F.V. James et al., Phys. Rev. A 64, 052312 (2001)

${ }^{23}$ R.P. Feynman, Int. J. Theor. Phys. 21, 467 (1982)

${ }^{24}$ D. Deutsch, Proc. R. Soc. Lond. A, 425, 73-90 (1989)

${ }^{25}$ For a review, R.B. Liu et al. Advances in Phys. 59, 703 (2010).

${ }^{26}$ A. Mohan et al., Appl. Phys. Lett. 98, 253102 (2011).

${ }^{27}$ Luttinger, J.M. Phys Rev. Lett. 102, 1030 (1956)

${ }^{28}$ O. Benson et al. Phys. Rev. Lett. 84, 2513 (2000)

${ }^{29}$ R. Oulton et al., Opt. Express 15, 17221 (2007). E. Gallardo et al., Opt. Express 18, 13301 (2010).

${ }^{30}$ M. Winger et al., Phys. Rev. Lett. 103, 207403 (2009) 